\title{
Circulation Studies in Experimental Phlegmasia Cerulea Dolens
}

\author{
THOMAS R. WEBER, M.D., CLAUDIO A. SALLES, M.D., \\ AND S. MARTIN LINDENAUER, M.D. \\ Department of Surgery, University of Michigan \& Vascular Research Laboratory, \\ Veterans Administration Hospital, Ann Arbor, Michigan 48105
}

Submitted for publication January 3, 1976

Phlegmasia cerulea dolens, or massive venous occlusion leading to circulatory tissue compromise, is a rare but serious disease which is associated with considerable morbidity and mortality $[5,7]$. The optimal treatment for phlegmasia is controversial, not only because the disease is uncommon, limiting the experience of any one group of surgeons, but also because the lack of an acceptable animal model has restricted studies of the basic pathophysiology of the disease [6]. Uncertainty concerning the pathophysiology of phlegmasia has led to a variety of therapeutic regimens, few of which seem to have a sound scientific basis.

Previous studies in this laboratory [8] have shown that venous ligation in the canine hind limb, followed by intravenous injection of thrombin, produces massive venous occlusion which leads to tissue necrosis and a clinical picture similar to human phlegmasia. In these studies we showed that massive venous occlusion resulted in markedly decreased tissue blood flow measured by the ${ }^{133} \mathrm{Xe}$ washout technique [8].

The present study utilizes this same model of phlegmasia in the canine hind limb to study arterial blood flow and arteriovenous shunting after venous obstruction. An aggressive surgical approach is suggested for the patient with phlegmasia cerulea dolens.

\section{METHODS}

\section{Experimental Model}

Adult, conditioned, mongrel dogs, of either sex, weighing between 15 and $25 \mathrm{~kg}$ were used throughout the study. They were anesthetized with intravenous sodium thiamylal and intubated. Anesthesia was maintained with small intermittent doses of sodium thiamylal, and each dog received $2000 \mathrm{ml}$ of Ringer's lactate solution during the operation. One femoral artery and vein were exposed in each dog through a longitudinal incision in the groin under aseptic conditions. The femoral vein and all tributaries joining the femoral vein just below the inguinal ligament were ligated. Immediately after ligation, 1000 USP units of thrombin were injected intravenously in a distal, dorsal vein in the paw. This preparation invariably leads to swelling in the hind limb which reaches massive proportions in $24 \mathrm{hr}$. In addition, bluish discoloration, ecchymoses, and eventual frank skin and muscle necrosis occurs in all untreated limbs. Ligation of the venous system in the canine hind limb without thrombin injection results in mild extremity swelling which resolves in $24-48 \mathrm{hr}$ and does not result in tissue necrosis [8].

\section{Circulatory Measurements}

Femoral artery flow was monitored with an electromagnetic flowmeter (Statham Instruments, Inc., Oxnard, Calif.) just prior to venous ligation and for approximately $\mathrm{l} \mathrm{hr}$ after the induction of phlegmasia. In addition, femoral artery flow was evaluated at 2 , 7 , and in surviving dogs, 30 days post-occlusion. In those groups in which therapy was attempted 1 day post-occlusion, femoral artery flow was measured immediately after treatment.

Muscle blood flow was measured by the ${ }^{133} \mathrm{Xe}$ washout technique, which has been described in detail in previous publications [3, 8]. Briefly, the disappearance of ${ }^{133} \mathrm{Xe}$ dissolved in saline from a muscle bed is 
directly related to the nutrient (capillary) blood flow to the bed, which in turn is a reflection of the arterial blood flow [3] and venous pressure [8] present in the muscle bed at the time of injection. Five hundred microcuries of ${ }^{133} \mathrm{Xe}$ is injected into an anterior tibial muscle, and the disappearance of the radioactivity is monitored by an external scintillation detector, The half-time ( $T 1 / 2)$ of the disappearance curve is calculated and substituted in the formula [3] to yield:

muscle blood flow $(\mathrm{ml} / 100 \mathrm{~g}$ muscle $/ \mathrm{min})=$ $48.51 / T 1 / 2$.

Xenon 133 washout curves were obtained just before venous occlusion, immediately after occlusion, and at 2 and 7 days postocclusion. In dogs undergoing treatment, muscle blood flow was also measured immediately after institution of treatment.

Arteriovenous (AV) shunting in the hind limb was measured by the method of LopezMajano et al. [2]. Technetium 99 labeled albumin microspheres (3M Co., St. Paul, Minn.), $15-25 \mu \mathrm{m}$ in diameter, were injected directly into the femoral artery. Microspheres passing through AV shunts were trapped in pulmonary capillaries, and measured by an external scintillation counter placed over the right chest. Following this, microspheres were injected intravenously to derive a value representing $100 \%$ shunting [2]:

$$
\begin{aligned}
\% \text { shunt }= & (\mathrm{cpm} / \mathrm{mCi} \text { injected ia } \times 100) / \\
& (\mathrm{cpm} / \mathrm{mCi} \text { injected iv })
\end{aligned}
$$

where $\mathrm{mCi}=$ millicuries of radioactivity contained in the intravenous (iv) and intraarterial (ia) doses, and cpm = counts per minute measured by the scintillation counter placed externally over the right chest. Arteriovenous shunt determinations were made just prior to venous occlusion and at 2 and 7 days post-occlusion. Dogs surviving for 30 days underwent $A V$ shunt determinations at that time.

\section{Experimental Groups}

Sixteen dogs underwent induction of phlegmasia, and were divided into 4 groups:
Group 1: (4 dogs) received no treatment.

Group 2: (4 dogs) were treated with systemic heparin, 100 units $/ \mathrm{kg} / 8 \mathrm{hr}$, starting 24 $\mathrm{hr}$ after the induction of phlegmasia and continuing for 7 days post-occlusion.

Group 3: (4 dogs) underwent bilateral lumbar sympathectomy 24 hours postvenous occlusion.

Group 4: (4 dogs) underwent iliofemoral venous thrombectomy with a fogarty catheter $24 \mathrm{hr}$ after venous occlusion. These dogs also received systemic heparin postthrombectomy as in Group 2.

\section{RESULTS}

Within $30 \mathrm{~min}$ after venous ligation and thrombin injection, all limbs demonstrated moderate to severe swelling, with bluish, ecchymotic discoloration. All limbs in Groups 1 (untreated) and 3 (sympathectomy) progressed to frank gangrene, with massive skin and muscle necrosis. These limbs remained massively swollen throughout the period of observation. Three of the 4 limbs in Group 2 (heparin) likewise rapidly progressed to gangrene, while the fourth limb in Group 3 returned to normal. In contrast, all limbs in Group 4 (thrombectomy and heparin) recovered completely, with marked improvement evident as early as $24 \mathrm{hr}$ postthrombectomy.

The results of the femoral artery flow measurements are shown in Table 1. The mean pre-occlusion femoral artery flow for all 16 dogs in the study was $133.4 \mathrm{ml} / \mathrm{min}$. Venous ligation and injection of thrombin was associated with an immediate and significant (compared to control) reduction in the femoral artery flow in all limbs in each group. However, 2 days post-occlusion (1 day posttreatment in Groups 2, 3, and 4), the femoral artery flow was within normal limits despite a clinical picture of rapidly progressing gangrene in Groups 1, 2, and 3. Seven days post-occlusion, all femoral artery flows were significantly increased compared to control despite frank gangrenous changes in the limbs in Groups 1, 2, and 3. However, the femoral artery flow returned to normal 30 days post-occlusion in Group 4. 
TABLE 1

Mean Femoral Artery Flows (cc/min) Determined at Various Intervals following Venous Occlusion

\begin{tabular}{cccccc}
\hline & \multirow{2}{*}{$\begin{array}{c}\text { Control } \\
\text { Group }\end{array}$} & \multicolumn{4}{c}{ Post-occlusion (days) } \\
\cline { 3 - 6 } (pre-occlusion) & Immediately & \multicolumn{1}{c}{2} & 7 & 30 \\
\hline 1 & 133.4 & $19.8^{*}$ & 148.8 & $380.0^{*}$ & \\
2 & 133.4 & $32.5^{*}$ & 130 & $520.0^{*}$ & \\
3 & 133.4 & $24.0^{*}$ & 117.5 & $545.0^{*}$ & 138.8 \\
4 & 133.4 & $22.5^{*}$ & $182.5^{*}$ & $245.0^{*}$ & \\
\hline
\end{tabular}

${ }^{*} P<0.05$ compared to control.

TABLE 2

Mean Muscle Blood Flow (ml/100 g muscle/min) Measured by ${ }^{133} \mathrm{Xe}$ Washout at Intervals following Venous Occlusion

\begin{tabular}{ccccc}
\hline & \multirow{2}{*}{$\begin{array}{c}\text { Control } \\
\text { Group }\end{array}$} & \multicolumn{3}{c}{ Post-occlusion (days) } \\
\cline { 3 - 5 } & 11.7 & Immediately & 2 & 7 \\
\hline 1 & 11.7 & $0.75^{*}$ & $2.6^{*}$ & $3.5^{*}$ \\
2 & 11.7 & $0.62^{*}$ & $3.5^{*}$ & $4.7^{*}$ \\
3 & 11.7 & $0.52^{*}$ & $2.2^{*}$ & $2.2^{*}$ \\
4 & $0.50^{*}$ & 12.3 & 11.4 \\
\hline
\end{tabular}

$* P<0.05$ compared to control.

The muscle blood flow determinations by ${ }^{133} \mathrm{Xe}$ washout are shown in Table 2 . The control value is the mean pre-occlusion muscle blood flow for 16 dogs. Venous occlusion promptly and significantly (compared to control) reduced muscle blood flow in all limbs. The muscle blood flow in Groups 1, 2, and 3 remained significantly lower than control values at 2 and 7 days post-occlusion. In contrast, muscle blood flow in Group 4 returned to normal 1 day post-thrombectomy, and remained at the level at 7 days postocclusion.

The results of the AV shunt determinations are tabulated in Table 3. The mean control $\Lambda \mathrm{V}$ shunt value was $10.1 \%$. This was increased significantly compared to control in all groups 2 days post-occlusion. The AV shunt measured 7 days post-occlusion re- mained significantly increased in Groups 1, 2, and 3, while in Group 4 this value was not significantly different from control. The percentage AV shunt measured 30 days postocclusion remained high, but was not significantly increased over the control value.

\section{DISCUSSION}

In most instances, the exact etiology of human phlegmasia is not known. However, almost all cases exhibit the rather abrupt onset of massive venous occlusion in the lower extremity $[1,4]$. When left untreated, phlegmasia progresses to gangrene in a large number of instances, and unfortunately this occurs even in $30-50 \%$ of patients aggressively treated [5].

There is little disagreement in the literature that anticoagulation with intravenous

TABLE 3

Mean Arteriovenous Shunt (\%) Determined at Intervals following Venous Occlusion

\begin{tabular}{ccccc}
\hline & Control & \multicolumn{3}{c}{ Post-occlusion (days) } \\
\cline { 3 - 5 } Group & 10.1 & 2 & 7 & 30 \\
\hline 1 & 10.1 & $41.3^{*}$ & $34.2^{*}$ & \\
2 & 10.1 & $35.4^{*}$ & $29.8^{*}$ & \\
3 & 10.1 & $46.6^{*}$ & $35.6^{*}$ & 21.8 \\
4 & & $29.4^{*}$ & 23.5 & \\
\hline
\end{tabular}

${ }^{*} P<0.05$ compared to control. 
heparin and bedrest are the main stays of therapy for phlegmasia, but when these measures do not result in improvement of the patient, there is confusion regarding additional therapeutic modalities. Part of this uncertainty is due to the lack of an adequate experimental model of phlegmasia to evaluate proposed therapeutic measures. In addition, objective information concerning large and nutrient vessel arterial flows are important in understanding the pathophysiology of phlegmasia and assessing the effectiveness of various modes of therapy.

The present study utilizes a combination of venous ligation and intravenous thrombin to produce an experimental model of phlegmasia that appears very similar to the human disease. The induction of phlegmasia in this way produces an immediate and significant drop in both large vessel (femoral artery) and nutrient (capillary) flow. Subsequently, in Groups 1, 2, and 3 (untreated, heparin alone, and sympathectomy) there was a dramatic increase in femoral artery flow while inappropriately the muscle blood flow remained low and the limbs became gangrenous. This suggested that blood was being shunted away from the capillary bed, and indeed the measurement of AV shunting in the limb indicated a 3- to 4-fold increase in AV shunt flow. It would appear that many of the venous collaterals which develop after massive venous occlusion reflect non-nutritive AV shunting.

In sharp contrast, all Group 4 limbs (venous thrombectomy plus heparin) recovered completely with no muscle necrosis and only minor skin breakdown which healed rapidly. There was also a rapid return of the muscle blood flow to normal 1 day post-thrombectomy, and normal AV shunt values determined 7 days post-thrombectomy.

Other potential modes of therapy for phlegmasia, such as fasciotomy, vasoactive pharmacologic agents, and thrombolytic agents are presently being evaluated using this same model.

Based on the data presented, it would appear that if bed rest and systemic heparin fail to significantly improve a patient with phlegmasia cerulea dolens, venous thrombectomy in addition to continued heparin might be the next logical choice of therapy. Sympathectomy or heparin alone do not improve nutrient blood flow in experimental phlegmasia. The largest shunt values and the lowest muscle flows were measured following sympathectomy. The role of other therapeutic modalities in the treatment of phlegmasia should be carefully evaluated in terms of blood flow measurements.

\section{SUMMARY}

A model of phlegmasia cerulea dolens in the canine hind limb was used to study the effects of heparin, sympathectomy, and venous thrombectomy plus heparin on limb hemodynamics. Only thrombectomy plus heparin restored nutrient blood flow and was associated with clinical recovery of the limb. This experimental model of phlegmasia appears to be an excellent tool for testing additional modalities of therapy for this serious disease.

\section{REFERENCES}

1. Brockman, S. K., and Varco, R. S. The pathologic physiology of phlegmasia cerulea dolens. Surgery 59:997, 1966.

2. Lopez-Majano, V., Rhodes, B., Wagner, H. Arteriovenous shunting in extremities. J. Appl. Physiol. 27:782, 1969.

3. Miller, T., Lindenauer, S. M., and Pozderac, R. V. Xenon ${ }^{133}$ clearance in the diagnosis of arterial occlusive disease. J. Surg. Res. 16:412, 1974.

4. Savage, J. P. The role of reflex spasm in the pathogenesis of venous ischemia. Surg. Gynecol. Obstet. 113:47, 1961.

5. Stallworth, J. M., Bradham, G. B., Kletke, R. R. and Price, R. G. Phlegmasia cerulea dolens: a 10 year review. Ann. Surg. 161:802, 1965.

6. Stallworth, J. M., Najib, A., Kletke, R., and Ramirez, A. Phlegmasia cerulea dolens: an experimental study. Ann. Surg. 165:860, 1967.

7. Veal, J., Dugan, T., Jamison, W., and Bauersfeld, R. Acute massive venous occlusion of the lower extremities. Surgery 29:355, 1951.

8. Weber, T., Lindenauer, S. M., Pozderac, R. V., Freedman, W., and Fry, W. J. Venous occlusion and Xenon $^{133}$ muscle clearance. Surg. Forum 25:260, 1974. 